\title{
Breastfeeding of mothers attending the outpatient clinic of Reina Sofia University Hospital (Cordoba, Spain)
}

\author{
Mohamed Farouk Allam ${ }^{1,2}$ \\ ${ }^{1}$ Department of Preventive Medicine and Public Health, Faculty of Medicine, University of Cordoba, Cordoba, Spain \\ ${ }^{2}$ Department of Community, Environmental and Occupational Medicine, Faculty of Medicine, Ain Shams University, Cairo, Egypt \\ Correspondence: Mohamed Farouk Allam, Department of Community, Environmental and Occupational Medicine, Faculty of \\ Medicine, Ain Shams University. 11566 Abbasia, Cairo, Egypt; \\ E-mail: fm2faahm@gmail.com and farouk.allam@ @ed.asu.edu.eg; Phone/Fax: +(202) 24346888; Mobile: + (2) 01143559946
}

\begin{abstract}
Background: Although the beneficial effects of breastfeeding are well-known, the prevalence of breastfeeding in many developed countries is quite low. Objective: The objective of this study was to estimate the prevalence of breastfeeding in the catchment area of Reina Sofia University Hospital of Cordoba (Spain). Materials and Methods: A cross sectional study was conducted for mothers attending the outpatient clinic of Reina Sofia University Hospital of Cordoba, for follow-up after 6 weeks of delivery. All mothers attending the outpatient clinic over 3 months accepted to participate in the study. Finally, we had a sample of 91 mothers. Study variables were age, educational level, gestational weeks, type of delivery, gender and weight of the new born, type of feeding for the baby over the first 6 weeks of his/her life, motives for abandoning breastfeeding, and motives for selecting the artificial formula. The information was collected through a previously validated interview questionnaire. Results: The mean age of the mothers was 31.8 (SD 3.13) years old. About 67\% of mothers selected exclusive breastfeeding and $4.39 \%$ selected the combination of breastfeeding with the artificial formula. More than $28 \%$ of mothers selected the artificial formula. The reasons for selecting the artificial formula were own decision (46.15\%), medical recommendation (11.53\%), lack of breast milk (3.84\%). The remaining $23.1 \%$ of mothers who selected the artificial formula refused to answer this question. Conclusions: Our results show that only two-third of mothers adopted breastfeeding in the first 6 weeks after delivery. Health education and promotion programmes for breastfeeding are urgently needed.
\end{abstract}

Keywords: Breastfeeding, Artificial Formula, Cross sectional, Cordoba, Spain.

\section{INTRODUCTION}

The World Health Organization (WHO) recommends exclusive breastfeeding in the first 6 months of life ${ }^{(1,2)}$.

Several studies showed short and long terms beneficial effects on health of new-borns and mothers (2). Early beneficial effects include lowering mortalities in premature new-borns ${ }^{(2,3)}$ and lowering infantile morbidities due to gastrointestinal tract, respiratory and urinary tract infections, together with atopic skin diseases ${ }^{(4,5,6,7)}$.

Also, many investigations showed that exclusive breastfeeding is associated with low rates of communicable disease in the first 6 months of life ${ }^{(8,9,10)}$.

The beneficial health effects of breastfeeding for new-borns explain the national and international supports to initiate and continue breastfeeding for all new-borns ${ }^{(1,3,11,12,13)}$.

Although the beneficial effects of breastfeeding are well-known, the prevalence of breastfeeding in many developed countries is quite low. In England, breastfeeding initiation was $72 \%$ and exclusive breastfeeding at 6-8 weeks decreased to $32 \%$. These results were obtained from a study conducted at 151 primary care trusts in England 2010-2011 ${ }^{(14)}$.

In the United States of America (USA), about $81.1 \%$ of infants born in 2013 had breastfeeding in the first 4 weeks of their lives. This prevalence decreased to $51.8 \%$ at 6 months and to $30.7 \%$ at 12 months ${ }^{(12)}$.

In a recent study conducted in Cyprus, about $84.3 \%$ of mothers initiated breastfeeding after delivery and before hospital discharge. This prevalence deceased at the sixth month to $32.4 \%$, with the highest decrease reported between the first and fourth months ${ }^{(15)}$.

Many mothers agree to breastfeed their new-borns during only a limited period of time and others do not even initiate breastfeeding. There are many reported factors which could influences in the early of cessation of breastfeeding. However, many studies showed that many mothers feel disappointed because they did not continue breastfeeding for longer periods ${ }^{(16,17,18,19)}$.

Based on the results of the previous studies, it is clear that it is necessary to evaluate the current situation of the breastfeeding in Andalusia in general and in Cordoba in particular. So, the objective of this study is to estimate the prevalence 
of breastfeeding in the catchment area of Reina Sofia University Hospital of Cordoba (Spain).

\section{MATERIAL AND METHODS}

Type of study: Cross sectional study.

Study setting: Reina Sofia University Hospital of Cordoba is a tertiary referral centre with 1500 beds, fully equipped to receive trauma and emergency patients. In addition, hospital activities extend to include organ and bone marrow transplantations. Owning to its location in the centre of Andalusia, it serves over one million persons.

Study period: From September 16, 2015 to December 15, 2015.

Study population: Mothers attending the outpatient clinic of Reina Sofia University Hospital of Cordoba, for follow-up after 6 weeks of delivery.

Inclusion criteria: Mothers of a 6 weeks aged baby attending the follow-up clinic of Reina Sofia University Hospital of Cordoba. Mothers of babies who did not need admission at neonatal intensive care unit (ICU) after delivery over the first 6 weeks of their lives. Accept to participate in the study.

Exclusion criteria: Mothers attending the out-patient clinic for any other reason, other than follow-up. Mothers of babies who needed admission at neonatal ICU. Mothers with human immunodefcieny virus (HIV). Mothers with any pathology in both breasts that impede breastfeeding.

Sample size: All mothers attending the outpatient clinic over the 3 months accepted to participate in the study. Finally, we had a sample of 91 mothers.

Ethical Considerations: Approval for this study was obtained by the institutional review board, and Ethical committee. All participants will sign an informed consent after explaining them the objective of the study.

Study variables: Age, educational level, gestational weeks, type of delivery, gender and weight of the new born, type of feeding for the baby over the first 6 weeks of his/her life, motives for abandoning breastfeeding, and motives for selecting artificial formula.

Data collection: The information was collected through interview questionnaire in Spanih.
This questionnaire included closed questions about the study variables.

Questionnaire: The questionnaire used in the study was previously validated and published in $2012^{(20)}$.

Statistical analysis: Data entry screen was built using Microsoft Excel 2010. Data was checked, coded and entered on the computer. First, descriptive analysis was done: frequency, percent, mean and standard deviation (SD). Thereafter, comparisons were carried out using the Student's ttest for continuous variables and Pearson's Chisquare test for categorical variables. Level of significance was set at $\mathrm{p}<0.05$. Analysis of the data was done using EPIDAT 4.2.

\section{RESULTS}

The mean age of the mothers was 31.8 (SD 3.13) years old and ranged from 23 to 42 . Only 17 (18.7\%) mothers had basic educational level (primary and preparatory schools) [Figure 1].

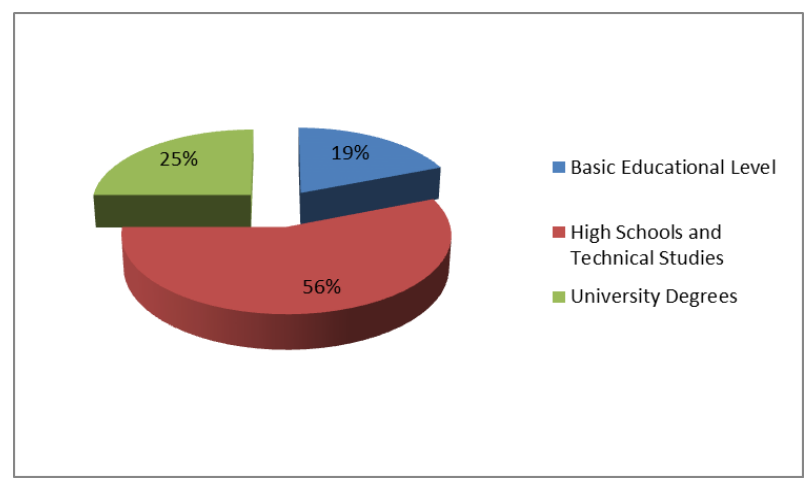

Figure (1): Educational level of participated mothers.

The mean gestational age was 40.8 (SD 1.4) weeks and ranged from 36 to 45 . Most of deliveries were vaginal deliveries $(70.3 \%)$ and caesarean sections were carried out in only $29.7 \%$ of deliveries. About $48 \%$ of new-borns were females, and the mean weight on birth was 3.402 (SD 0.39) Kg.

More than one-fourth of the participated mothers selected the artificial formula (Table 1). The reasons for selecting the artificial formula were own decision (46.15\%), medical recommendation $(11.53 \%)$, lack of breast milk (3.84\%). The remaining $23.1 \%$ of mothers who selected the artificial formula refused to answer this question. 
Table (1): Feeding of new-borns in the first 6 weeks after delivery according to mothers attending the outpatient clinic of Reina Sofia University Hospital of Cordoba (Spain).

\begin{tabular}{|l|c|c|}
\hline \multicolumn{1}{|c|}{ Type of Feeding } & Number & Percentage \\
\hline Exclusive Breastfeeding & 61 & 67 \\
\hline Mixed: Breastfeeding and Artificial Formula & 4 & 4.4 \\
\hline Exclusive Artificial Formula & 26 & 28.6 \\
\hline Total & 91 & 100 \\
\hline
\end{tabular}

Age of mothers who adhered to exclusive breastfeeding (mean 31.9, SD 3.44 years) compared with that of mothers who shifted to exclusive artificial formula or mixed feeding (mean 31.6, SD 2.43 years) showed no statistical significant difference (Student's t-test: 0.34 , p value: 0.668 ).

No statistical significant difference was found between exclusive breastfeeding and exclusive artificial formula or mixed feeding mothers regarding the educational levels (table 2).

Table (2): Comparative analysis between exclusive breastfeeding and exclusive artificial formula or mixed feeding mothers regarding the educational levels.

\begin{tabular}{|c|c|c|}
\hline $\begin{array}{c}\text { Educational Level of } \\
\text { Mothers }\end{array}$ & $\begin{array}{c}\text { Exclusive } \\
\text { Breastfeeding }\end{array}$ & $\begin{array}{c}\text { Exclusive Artificial } \\
\text { Formula or Mixed } \\
\text { Feeding }\end{array}$ \\
\hline $\begin{array}{c}\text { Basic Educational } \\
\text { Levels }\end{array}$ & $12(19.7 \%)$ & $5(16.6 \%)$ \\
\hline $\begin{array}{c}\text { High Schools and } \\
\text { Technical Studies }\end{array}$ & $15(24.6 \%)$ & $8(26.7 \%)$ \\
\hline University Degrees & $34(55.7 \%)$ & $17(56.7 \%)$ \\
\hline Total & $61(100 \%)$ & $30(100 \%)$ \\
\hline
\end{tabular}

Pearson's Chi-square test: 0.135 (p-value 0.935)

No association was found between infants' feeding (exclusive breastfeeding vs exclusive artificial formula or mixed feeding) and mode of delivery (table 3 ).

Table (3): Comparative analysis between exclusive breastfeeding and exclusive artificial formula or mixed feeding mothers regarding the mode of delivery.

\begin{tabular}{|l|c|c|}
\hline \multicolumn{1}{|c|}{ Mode of Delivery } & $\begin{array}{c}\text { Exclusive } \\
\text { Breastfeeding }\end{array}$ & $\begin{array}{c}\text { Exclusive Artificial } \\
\text { Formula or Mixed } \\
\text { Feeding }\end{array}$ \\
\hline Vaginal Delivery & $42(68.9 \%)$ & $22(73.3 \%)$ \\
\hline Caesarean Section & $19(31.1 \%)$ & $8(26.7 \%)$ \\
\hline Total & $61(100 \%)$ & $30(100 \%)$ \\
\hline
\end{tabular}

Pearson's Chi-square test: 0.194 (p-value 0.660)

\section{DISCUSSION}

Over the last decades, the WHO insisted on the importance of breastfeeding for reducing infant mortality rates and morbidities in the first two years of life ${ }^{(13)}$. The current study evaluated the prevalence of breastfeeding in the catchment area of Reina Sofia University Hospital of Cordoba in only the first 6 weeks of life.

Before reaching conclusions based on the results of the current study, we should consider a number of potential limitations. The study included a small sample size (91 mothers) and calculating the prevalence of breastfeeding in Cordoba might need a larger sample size. However, the study included all mothers attending the outpatient clinic of Reina Sofia University Hospital of Cordoba, over 3 months after 6 weeks of delivery. All these mothers accepted to participate in the study and answered all relevant questions to the main objective of the study.

The results of the study were quite disappointing; only two-third of interviewed mothers adhered to exclusive breastfeeding in the first 6 weeks after delivery. Our results raise a very important question: To what extent would further follow-up of these mothers, for 6 or even 12 months, might lower their adherence to exclusive breastfeeding?

In a recent cohort study conducted in Madrid, about $64.4 \%$ of participated women (total 523) maintained exclusive breastfeeding after 3 months of delivery. This figure declined markedly to $31.4 \%$ after 6 months of delivery ${ }^{(17)}$.

In a follow-up study conducted in Canada, exclusive breastfeeding rates during the first 6 months of life decreased gradually from $63.6 \%$ after 1 month, to $49.6 \%$ after 3 months, and to $13.8 \%$ after 6 months ${ }^{(21)}$.

In a recent population based follow-up study conducted in Italy, exclusive breastfeeding prevalence rate were $57.2 \%$ at discharge, $48.6 \%$ at 3 months, and $5.5 \%$ at 6 months ${ }^{(22)}$.

It is clear from previous studies that exclusive breastfeeding decreases progressively to very low rates after 6 months of delivery. Thus exclusive breastfeeding rate in our study $(67 \%)$ is expected to decrease progressively.

In Spain, follow-up for women after 6 weeks of delivery is usually conducted in the Family Medicine Centers; It is recommended that new studies about breastfeeding in Spain to be conducted in the Family Medicine Centers to facilitate follow-up for long periods. 
In the present study, medical recommendation and lack of breast milk accounted for less than $16 \%$ for discontinuing of breastfeeding and shifting to artificial formula. This agrees with the results of a previous study conducted in Canada, which showed that $21.6 \%$ of interviewed mothers stopped breastfeeding due to concerns about milk supply ${ }^{(23)}$.

Recent systematic review including 27 articles showed clearly that lack of breast milk had low level of evidence in breastfeeding cessation ${ }^{(24)}$.

The main reason for discontinuing of breastfeeding and shifting to artificial formula in the present study was the mothers' own decision (46.15\%). Of note, about $23.1 \%$ of interviewed mothers refused to give a reason for discontinuing of breastfeeding and shifting to artificial formula. It is clear that more than two-third of mothers who shifted to artificial formula had non-medical reasons for that.

In a cross sectional study conducted in Canada, interviewed mothers reported inconvenience or fatigue associated with breastfeeding (22.6\%) and need to return to work (20\%) as the mean reasons for stopping breastfeeding ${ }^{(23)}$.

Possible sociodemographic and physical risk factors associated with stopping breastfeeding include age of the mother, educational level, and mode of delivery ${ }^{(13,24)}$.

In the present study, age of the mother was not an associated risk factor with discontinuing exclusive breastfeeding. This agrees with a recent study conducted in Spain where 569 mothers were interviewed to investigate the barriers to maintain breastfeeding. This study showed that neither the age of the mother nor the parity was risk factor for stopping breastfeeding ${ }^{(25)}$.

Similar observations were reported by Mangrio and collaborators in their recent systematic review ${ }^{(24)}$.

The results of the present study showed no association between exclusive breastfeeding practice and the level of education. These results are in concordance with the results of a Spanish ecological study that evaluated breastfeeding trend over a 40 -year period ${ }^{(26)}$.

Data from a Regional Immunization Survey in Belgium with the participation of 525 mothers showed that educational level has no association with the duration of breastfeeding ${ }^{(27)}$.

In contrary to the results of the current study, data from the Nepal Demographic and Health
Surveys (2011) showed that early initiation of breastfeeding was higher among mothers with primary education and mothers with secondary or higher education compared to mothers with no education (28). Also in a previous cohort study conducted in Valdivia (Chile) on 315 mothers, it was found that maternal education years were associated with continuing of exclusive breastfeeding ${ }^{(29)}$.

These contradictory finding of the results of the different studies could be related to other factors like socioeconomic level, mother's employment, maternity leave duration, and social welfare support system ${ }^{(30,31)}$.

A systematic review published in 2011 suggested that breastfeeding education rather than the level of education is more effective in increasing breastfeeding duration, especially in low- and middle-income countries ${ }^{(32)}$.

The present study did not find any association between exclusive breastfeeding and the mode of delivery. A recent study conducted in Nicaragua showed that breastfeeding initiation and/or duration was not associated with the mode of delivery ${ }^{(33)}$. Meanwhile, the results of the Demographic and Health Survey in Pakistan (2011) showed that cesarean sections, compared with vaginal deliveries, were associated with more difficulties in initiating breastfeeding ${ }^{(34)}$.

Of no doubt, the small sample size of the present study might not help to identify with precision the main reasons for the non-adherence of one-third of participants to exclusive breastfeeding.

\section{CONCLUSION}

Exclusive breastfeeding in the first 6 weeks in our area is relatively low. Health education programs for both mothers and healthcare professionals are needed, together with breastfeeding policies and supporting plans.

\section{REFERENCES}

\section{World Health Organization. Infant and young child feeding (2003): A tool for assessing national practices, policies and programmes. Geneva: WHO, 2003. Available at: http://www.who.int/child-adolescenthealth/ New_Publications/ NUTRITION/ icyf.pdf}
2. Mathur NB, Dhingra D (2014): Breastfeeding. Indian J Pediatr., 81(2):143-9. 
3. Binns CW, Lee MK (2014): Exclusive breastfeeding for six months: the WHO six months recommendation in the Asia Pacific Region. Asia Pac J Clin Nutr., 23(3):344-50.

4. Kramer MS, Kakuma R (2012): Optimal duration of exclusive breastfeeding. Cochrane Database Syst Rev., (8):CD003517.

5. Comité de nutrition de la Société française de pédiatrie, Turck $D$, Vidailhet $M$, Bocquet A, Bresson JL, Briend A, Chouraqui JP, Darmaun D, Dupont C, Frelut ML, Girardet JP, Goulet O, Hankard R, Rieu D, Simeoni U (2013): Breastfeeding: health benefits for child and mother. Arch Pediatr., 20 (2):S29-48.

6. Shamir R (2016): The Benefits of Breast Feeding. Nestle Nutr Inst Workshop Ser., 86:67-76.

7. Oddy WH (2017): Breastfeeding, Childhood Asthma, and Allergic Disease. Ann Nutr Metab., 70 (2):26-36.

8. Hanson LA (1998): Breastfeeding provides passive and likely long-lasting active immunity. Ann Allergy Asthma Immunol., 81(6):523-33.

9. Oddy WH (2001): Breastfeeding protects against illness and infection in infants and children: a review of the evidence. Breastfeed Rev., 9(2):11-8.

10. Quigley MA, Carson C, Sacker A, Kelly Y (2016): Exclusive breastfeeding duration and infant infection. Eur J Clin Nutr., 70(12):1420-1427.

11. Mårild S, Hansson S, Jodal U, Odén A, Svedberg K (2004): Protective effect of breastfeeding against urinary tract infection. Acta Paediatr., 93(2):164-8.

12. Centers for Disease Control and Prevention. Breastfeeding Rates Continue To Rise (2016): Available at: https:// www. cdc. gov/ media/ releases/ 2016/ p0822-breastfeeding-rates.html

13. World Health Organization (2017): 10 facts on breastfeeding. Updated August 2017. Available at: http:// www. who. int/features/factfiles/breastfeeding/en/

14. Oakley LL, Renfrew MJ, Kurinczuk JJ, Quigley MA (2013): Factors associated with breastfeeding in England: an analysis by primary care trust. BMJ Open, 3(6):e002765.

15. Economou M, Kolokotroni O, PaphitiDemetriou I, Kouta C, Lambrinou E, Hadjigeorgiou E, Hadjiona V, Tryfonos F, Philippou E, Middleton N (2018): Prevalence of breast-feeding and exclusive breast-feeding at $48 \mathrm{~h}$ after birth and up to the sixth month in Cyprus: the BrEaST start in life project. Public Health Nutr., 21(5):967-80.
16. Zanardo V, Cavallin F, Guerrini $P$, Bertin M, Straface G (2017): Prepregnancy Body Mass Index Shift Across Gestation: An Association with Breastfeeding Practices? Breastfeed Med., 12(10):615-20.

17. Fernández-Cañadas Morillo A, Durán Duque M, Hernández López AB, Muriel Miguel C, Martínez Rodríguez B, Oscoz Prim A, Pérez Riveiro P, Salcedo Mariña A, Royuela Vicente A, Casillas Santana ML, Marín Gabriel MA (2017): A Comparison of Factors Associated with Cessation of Exclusive Breastfeeding at 3 and 6 Months. Breastfeed Med., 12(7):430-5.

18. Cato K, Sylvén SM, Lindbäck J, Skalkidou A, Rubertsson C (2017): Risk factors for exclusive breastfeeding lasting less than two monthsIdentifying women in need of targeted breastfeeding support. PLoS One, 12(6):e0179402.

19. Bærug A, Laake P, Løland BF, Tylleskär T, Tufte E, Fretheim A (2017): Explaining socioeconomic inequalities in exclusive breast feeding in Norway. Arch Dis Child, 102(8):708-14.

20. Martín Ruiz MC, Brunete Aceña I, Ruiz Gutiérrez D, Ruiz Cobo J, Farouk Allam M (2012): Breastfeeding in a rural area: Situational Analysis [article in Spanish]. Medicina General y de Familia, 1(2):68-70.

21. Al-Sahab B, Lanes A, Feldman M, Tamim H (2010): Prevalence and predictors of 6-month exclusive breastfeeding among Canadian women: a national survey. BMC Pediatrics, 10:20.

22. Lauria L, Spinelli A, Grandolfo M (2016): Prevalence of breastfeeding in Italy: a population based follow-up study. Ann Ist Super Sanita., 52(3):457-61.

23. Brown CR, Dodds L, Legge A, Bryanton J, Semenic S (2014): Factors influencing the reasons why mothers stop breastfeeding. Can $\mathbf{J}$ Public Health, 105(3):e179-85.

24. Mangrio E, Persson $\mathrm{K}$, Bramhagen AC (2017): Sociodemographic, physical, mental and social factors in the cessation of breastfeeding before 6 months: a systematic review. Scand J Caring Sci., doi: 10.1111/scs.12489. 
25. Díaz-Gómez M, Ruzafa-Martínez M, Ares S, Espiga I, De Alba C (2016): [Motivations and Perceived Barriers to Initiate or Sustain Breastfeeding among Spanish Women]. Rev Esp Salud Publica, 90:e1-e18. Spanish.

26. Colodro-Conde L, Sánchez-Romera JF, Tornero-Gómez MJ, Pérez-Riquelme F, Polo-Tomás M, Ordoñana JR (2011): Relationship between level of education and breastfeeding duration depends on social context: breastfeeding trends over a 40-year period in Spain. J Hum Lact., 27(3):272-8.

27. Robert E, Coppieters Y, Swennen B, Dramaix M (2014): Breastfeeding Duration: A Survival Analysis-Data from a Regional Immunization Survey. BioMed Res Int., 2014;529790.

28. Acharya P, Khanal V (2015): The effect of mother's educational status on early initiation of breastfeeding: further analysis of three consecutive Nepal Demographic and Health Surveys. BMC Public Health, 15:1069-?.

29. Barría RM, Santander G, Victoriano $T$ (2008): Factors associated with exclusive breastfeeding at 3 months postpartum in Valdivia, Chile. J Hum Lact., 24(4):439-45.
30. Flacking R, Dykes F, Ewald U (2010): The influence of fathers' socioeconomic status and paternity leave on breastfeeding duration: a population-based cohort study. Scand J Public Health, 38(4):337-43.

31. Steurer LM (2017): Maternity Leave Length and Workplace Policies' Impact on the Sustainment of Breastfeeding: Global Perspectives. Public Health Nurs., 34(3):286-94.

32. Imdad A, Yakoob MY, Bhutta ZA (2011): Effect of breastfeeding promotion interventions on breastfeeding rates, with special focus on developing countries. BMC Public Health, 11(3):S24.

33. Kiani SN, Rich KM, Herkert D, Safon C, Pérez-Escamilla R (2018): Delivery mode and breastfeeding outcomes among new mothers in Nicaragua. Matern Child Nutr., 14(1).

34. Saeed G, Fakhar S, Imran T, Khawaja Abbas L (2011): The Effect of Modes of Delivery on Infants' Feeding Practices. Iran J Med Sci., 36(2):128-32. 